\title{
Percepciones, creencias y saberes asociados a las enfermedades cardiovasculares en un grupo de mujeres de La Pampa, Argentina
}

\section{Perceptions, beliefs and knowledge associated with cardiovascular diseases in a group of women of the La Pampa, Argentina}

Daniela Moyano ${ }^{1, a}$, Rosana del Sueldo ${ }^{2, b}$

1 Facultad de Ciencias Médicas, Universidad Nacional de Córdoba. Córdoba, Argentina.

2 Ministerio de Salud de la Provincia de La Pampa, Hospital José Padrós. La Pampa, Argentina.

a Magíster en Salud Pública. Licenciada en Nutrición, b Licenciada en Bioquímica

Correspondencia

Daniela Moyano

moyanodaniela12@gmail.com

Recibido: 20/11/2017

Arbitrado por pares

Aprobado: 20/12/2017

Citar como: Moyano D, Del Sueldo R. Percepciones, creencias y saberes asociados a las enfermedades cardiovasculares en un grupo de mujeres de La Pampa, Argentina. Acta Med Peru. 2017;34(4):294-300

\section{RESUMEN}

Objetivo: Explorar las percepciones, creencias y saberes de un grupo de mujeres adultas asociados a las enfermedades cardiovasculares (ECV) de La Pampa, Argentina. Material y métodos: Se llevó a cabo un estudio cualitativo de carácter exploratorio a partir de 15 entrevistas exploratorias a mujeres entre 30 a 60 años que se atendieron en el sistema de salud pública de la provincia de La Pampa, Argentina durante el 2014. Se conformaron dos grupos de análisis; uno constituido por mujeres que asistieron a una consulta externa y otro por participantes de un programa público de prevención de enfermedades no trasmisibles con foco en actividad física. Se utilizó un muestreo intencional y por oportunidad. Resultados: Las mujeres percibieron su estado de salud general y cardiovascular como "bueno", con cierto grado de desconocimiento sobre cuáles son las ECV y con las creencias que se deben a "herencia familiar" o "azar". Los saberes sobre factores de riesgo cardiovasculares se centraron en "colesterol elevado", y en menor medida "sedentarismo" y "alimentación poco saludable". El "sedentarismo" fue reconocido principalmente por mujeres bajo el programa preventivo. La mayoría no supieron reconocer ninguna conducta que beneficie el cuidado de la salud cardiovascular, mientras que las que sí reconocieron la existencia solo hicieron alusión a la actividad física y a la alimentación saludable. No se visualizaron creencias sobre el vínculo entre la "edad" y "ser mujer" con las ECV. Conclusiones: Las mujeres entrevistadas tuvieron algún grado de desconocimiento y una baja percepción del riesgo reforzadas por creencias arraigadas sobre las ECV y que podrían estar explicadas por diferentes determinantes sociales, culturales y de género.

Palabras clave: Mujeres; Enfermedades cardiovasculares; Salud pública (fuente: DeCS BIREME). 


\begin{abstract}
Objective: The study had it aim explorer the perceptions, beliefs and knowledge of a group adult women associated with cardiovascular diseases (CVD) in the context of the public health system of Argentina. 2014. Material and methods: A qualitative study of exploratory character was carried out from 15 exploratory interviews to women between 30 and 60 years old that were treated in the public health system if the La Pampa province, Argentina in the year 2014. Two analysis groups were formed, one constituted by women who attended an outpatient consultation by outpatient hospital and other group consisted of participants in a public program for the prevention of non-communicable chronic diseases with a focus on physical activity. The used a purposive and opportunity sampling approach. Results: The women perceived his general health and cardiovascular as "good", whit some degree of ignorance about which are cardiovascular diseases and with beliefs that are due to "family inheritance" or "random". The knowledge about cardiovascular risk factors they focused in "high cholesterol", to a less extent in "sedentarism" and "unhealthy eating". Mainly women under preventive program recognized the "sedentarism". Many of the interviewees did not recognize any behavior that benefits the care of cardiovascular health, while those who did recognize existence only alluded to physical activity and healthy eating. There were no beliefs of the link between "age" and "being a woman" with cardiovascular diseases. Conclusions: It is concluded that women had some degree of ignorance and a low perception of risk reinforced by beliefs rooted in CVD and that could be explained by different social, cultural and, gender determinants.

Keywords: Woman; Cardiovascular diseases; Public health (source: MeSH NLM).
\end{abstract}

\section{INTRODUCCIÓN}

La transición demográfica y epidemiológica de América Latina se ha caracterizado por procesos de cambios en los patrones de morbimortalidad de las poblaciones con una preocupante tendencia hacia el aumento de las enfermedades no transmisibles (ENT), entre las que se incluyen las cardiovasculares (ECV) ${ }^{[1-3]}$, siendo estas últimas la principal causa de muerte en Argentina ${ }^{[4]}$.

Según estudios epidemiológicos el riesgo de eventos de morbimortalidad por ECV se incrementa cuando confluyen varios factores de riesgo ${ }^{[5]}$ constituyendo una "modalidad de construcción del riesgo" [6]. Hoy se reconocen diversos factores asociados a estas enfermedades, siendo en su gran mayoría prevenibles y modificables, como hábitos alimentarios poco saludables, el sobrepeso, obesidad, hipertensión arterial, glucemia elevada, parámetros elevados de colesterol y triglicéridos, como también el sedentarismo y hábitos nocivos ${ }^{[7]}$, los cuales han venido en aumento durante las últimas décadas en Argentina ${ }^{[5]}$.

Existen además otros determinantes de las ECV provenientes de la esfera social y ambiental ${ }^{[5,8]}$, siendo el escenario en donde se contextualiza el riesgo en la salud y se interrelacionan con los físicos y biológicos para formar un panorama complejo en el cual se desarrolla el fenómeno epidemiológico. Si bien en el país desde la esfera pública se vienen realizando estudios con representatividad nacional sobre la prevalencia e incidencia de uno o más factores de riesgo para ENT ${ }^{[5]}$, se necesita mayor producción de evidencias desde la perspectiva de los actores que permitan rescatar los sentidos y procesos en el contexto de las enfermedades cardiovasculares.
Por otro lado, incorporar un enfoque de género en torno a las ECV parte de reconocer la existencia de modos diferenciales de vivir, enfermar y morir, entre hombres y mujeres donde la evidencia viene identificando algunos modos específicos relativos al género que se encuentran ligados a los ideales y exigencias diferenciales en la vida en sociedad, determinando en última instancia su capacidad para modificar comportamientos de riesgo; así como, el éxito de las intervenciones frente a estas enfermedades ${ }^{\left[{ }^{[9}\right.}$.

Si bien, la prevalencia de ECV se encuentran presente tanto en hombres como mujeres los factores de riesgo asociados como el tabaquismo, obesidad y consumo de alcohol vienen aumentando de manera más marcada en las mujeres ${ }^{[10]}$; donde además, se visualiza que presentan tasas más elevadas de complicaciones por infarto de miocardio ${ }^{[10]}$ y pueden ser menos propensas que los hombres a manifestar el dolor precordial como síntoma del infarto ${ }^{[11]}$.

Además, existen factores condicionantes que van exponiendo de manera diferenciada a cada grupo y definiendo las capacidades para modificar comportamientos de riesgo y de protección, donde frente a las ECV se despliegan múltiples prácticas de cuidado específicas y distintivas de género ${ }^{[9-15]}$. Por ejemplo, se observa que las mujeres acuden a los servicios de salud más tardíamente que los hombres ${ }^{[11]}$.

Por ello, el objetivo de estudio es explorar las percepciones, creencias y saberes de un grupo de mujeres adultas asociados a las enfermedades cardiovasculares (ECV) de La Pampa, Argentina. 


\section{MATERIAL Y MÉTODOS}

\section{Diseño de estudio}

El presente estudio fue de tipo cualitativo con enfoque exploratorio ${ }^{[16]}$, que exploró las percepciones, creencias y saberes de un grupo de mujeres adultas asociados a las enfermedades cardiovasculares tomando como base teórica el "Modelo de Creencias en Salud" (MCS) ${ }^{[17,18]}$.

\section{Participantes y muestreo}

La muestra quedó constituida por 15 mujeres adultas entre 30 a 60 años que formaron parte del sistema de salud pública de la Provincia de La Pampa, Argentina, durante el año 2014.

Se conformaron dos grupos de análisis: uno constituido por mujeres que asistieron a una consulta externo $(n=7)$ y el otro grupo estuvo conformado por mujeres que formaban parte de un programa provincial de prevención de ENT con foco en actividad física $(n=8)$.

La selección de las participantes se hizo por medio de un muestreo intencional ${ }^{[19]}$, y por oportunidad, según los siguientes criterios de inclusión: formar parte del sistema de salud pública de la provincia (asistencia al hospital público y/o programa preventivo); grupo etario (adulto); género (femenino); y presencia de factor/es de riesgo para ECV (tener por lo menos al momento de la entrevista uno de los siguientes factores: hipertensión arterial, diabetes, dislipemias, sobrepeso/obesidad).

\section{Recolección de datos}

La recolección de datos fue a través de entrevistas en exploratorias ${ }^{[20]}$, a partir de una guía de preguntas abiertas elaborada en base las dimensiones del "Modelo de Creencias en Salud" (MCS) ${ }^{[17,18]}$ entre los meses de mayo y noviembre del 2014 , en el marco de un acompañamiento en las diferentes actividades realizadas desde el programa y de presenciar las consultas externas.

Las dimensiones de análisis a partir del MCS ${ }^{[17]}$ fueron: severidad percibida, vulnerabilidad percibida, probabilidad de la acción preventiva y conductas percibidas. Las categorías emergentes de estas dimensiones se estructuraron de forma inductiva para integrarse en la formulación final de los resultados reportados.

\section{Análisis de datos}

La información cualitativa derivada del trabajo de campo se transcribió fielmente y se analizó de manera reflexiva por medio de la técnica análisis de contenido ${ }^{[21]}$. Las transcripciones textuales de las entrevistas se constituyeron en la unidad de análisis y se clasificaron y codificaron según los objetivos del estudio y las dimensiones abordadas.
Los análisis se llevaron a cabo utilizando el software ATLAS.ti (Scientific Software Development, Berlín, Alemania) combinado con la técnica manual de codificación de información. El análisis consistió en: 1) codificación de nodos amplios (dimensiones); 2) distinción de información específica para identificar elementos comunes, divergentes y emergentes (categorías emergentes); 3 ) comparación y condensación de la información.

Para mejorar la validez interna del estudio, se revisaron todas las transcripciones por lo menos dos veces. Además, se seleccionaron frases textuales para ilustrar el contenido central de cada categoría cuidando el anonimato.

\section{Aspectos éticos}

En el presente estudio se garantizaron todos los aspectos éticos correspondientes provenientes del estudio marco de esta investigación ${ }^{[22]}$, que fue aprobado por el Comité de Ética de Investigaciones en Salud del "Hospital Gobernador Centeno, Ministerio de Salud de la Provincia de La Pampa, Argentina. Los entrevistados consintieron su participación a través de la aceptación del consentimiento informado.

\section{RESULTADOS}

Las informantes fueron de género femenino $(n=15)$ y asistieron al sistema de salud pública durante el periodo de recolección de datos. Sus edades estuvieron comprendidas entre 30 y 60 años (Tabla 1).

A partir del MCS y las categorías emergentes surgió una propuesta de "marco conceptual de subjetividades" (Figura 1) a partir de los discursos reflejando cierta direccionalidad y relaciones entre las categorías emergentes y dimensiones.

\section{Severidad percibida}

Al indagar sobre la dimensión de severidad percibida en torno a las ECV no se encontraron ideas claras relacionadas a esta dimensión. Si bien las informantes no manifestaron que tan severa creen que son estas enfermedades, algunas de ellas anclaron sus percepciones a ciertas creencias sobre cuál podría ser el origen vinculado a la severidad; en ese sentido vincularon a la "herencia familiar" o que se debe solo al "azar". También se observó que, en el imaginario de algunas mujeres, la severidad de estas enfermedades se deben puramente a los "problemas de la vida cotidiana" durante el tránsito por la edad adulta.

Por otro lado, se exploró sobre algunas creencias sobre la relación entre las ECV y la edad. Solo una minoría que sí reconoció alguna relación entre estos dos aspectos, lo sustentaron a través de mecanismos emocionales o de estrés psicológico que se vivencian con mayor frecuencia en la edad adulta y donde según sus visiones se pueden vincular posteriormente con las enfermedades que afectan el corazón y su severidad. 
Tabla 1. Características clínicas y sociodemográficas de las mujeres entrevistadas ( $n=15)$.

\begin{tabular}{|c|c|c|c|c|c|c|}
\hline \multirow{2}{*}{ Variable } & \multicolumn{2}{|c|}{ Consultorio externo } & \multicolumn{2}{|c|}{ Programa preventivo } & \multicolumn{2}{|c|}{ Total } \\
\hline & $\mathbf{n}$ & $\%$ & $n$ & $\%$ & $n$ & $\%$ \\
\hline \multicolumn{7}{|l|}{ Sexo (\%) } \\
\hline Femenino & 7 & 45 & 8 & 55 & 15 & 100 \\
\hline \multicolumn{7}{|c|}{ Grupo etario (años, \%) } \\
\hline $30-49$ & 6 & 37 & 6 & 44 & 12 & 81 \\
\hline $50-60$ & 2 & 13 & 1 & 6 & 3 & 19 \\
\hline \multicolumn{7}{|c|}{$\begin{array}{l}\text { Presencia de factores de riesgos presentes } \\
\text { en mujeres* }\end{array}$} \\
\hline 1 & 6 & 37,5 & 6 & 37,5 & 12 & 75 \\
\hline $2-3$ & 2 & 13 & 1 & 6 & 3 & 19 \\
\hline 4 o más & 0 & 0 & 1 & 6 & 1 & 6 \\
\hline
\end{tabular}

* Se consideraron los siguientes factores de riesgo para ECV: hipertensión arterial, diabetes, dislipemias, sobrepeso/obesidad.

Solo una mujer que asistió al consultorio externo hizo referencia a que estas enfermedades, a no ser que sean congénitas, se dan a partir de los 40 años de edad.

"Si puede ser... pienso que la edad te lleva a tener más problemas..." (mujer, 50 años, hipertensa y obesa-Programa preventivo de actividad física).

"Depende...si son congénitas...pero la mayoría desde los 40 para arriba..." (mujer, 45 años, hipotiroidea-obesidad-Consultorio externo).

Por otra parte, respecto a las creencias vinculación entre la salud cardiovascular y el "ser mujer", la mayoría de informantes manifestaron no encontrar ningún mecanismo de vinculación, estas percepciones se vieron reflejadas en ambos grupos estudiados. Una minoría que si estableció alguna relación no logró fundamentar desde lo discursivo sobre esta premisa y solamente una entrevistada expresó que esta relación se podría asociar a la etapa de la menopausia propia de las mujeres (mecanismos biológicos).

"No...Salvo cuando entramos a la menopausia..." (mujer, 43 años, hipotiroidea-artritis- Programa preventivo de actividad física).

\section{Vulnerabilidad percibida}

Cuando se indagó sobre la vulnerabilidad de su estado de salud, las informantes señalaron percibir su estado de salud general como "bueno" o "muy bueno" donde en algunos casos dentro del grupo que asistió al programa preventivo manifestaron ciertas sensaciones de "bienestar" en su salud y que esta se encontraba vinculada con la práctica de actividad física.

"Bastante bien... Me noto activa..." (mujer, 48 años, diabética, obesa e hipertensa-Programa preventivo de actividad física).
Solamente las mujeres que manifestaron percibir su salud general "más o menos" o "malo" se relacionó con sensaciones de "malestar" a causa de transitar otra enfermedad crónica asociada a dolores (ej. artritis reumatoidea).

"Bastante mal... porque siempre tengo algún dolor..." (mujer, 50 años, hipertensa, artritis reumatoidea-Programa preventivo de actividad física).

Al consultar sobre el estado de salud cardiovascular, todas las entrevistadas la percibieron como "buena", no considerando la presencia de factores riesgo asociados ya adquiridos y en varios casos estas afirmaciones se respaldaron sobre algunos mecanismos condicionantes como "me lo dijo el cardiólogo" o haciendo alusión a alguna práctica médica "por qué me hice el electro" o bajo la idea de "soy saludable".

También se observaron algunas manifestaciones y sensaciones de "vitalidad" en mujeres que se encontraban formando parte del programa preventivo asociadas a su salud cardiovascular.

\section{Probabilidad de la acción preventiva}

Al analizar la probabilidad de la acción preventiva, se encontraron ciertas barreras relacionadas a un bajo caudal de saberes específicos sobre las enfermedades cardiovasculares. Se pudo observar que una parte importante de las mujeres manifestó un desconocimiento de cuáles eran las enfermedades que afectaban su sistema cardiovascular, sus consecuencias y sus formas de prevención. Este desconocimiento estuvo presente en ambos grupos bajo estudio.

En una pequeña parte que refirió conocer algunas de estas afecciones, también se encontraron presentes barreras al momento de identificar estas enfermedades; observándose que, en muchos casos se tendió a confundirlas con sus factores 

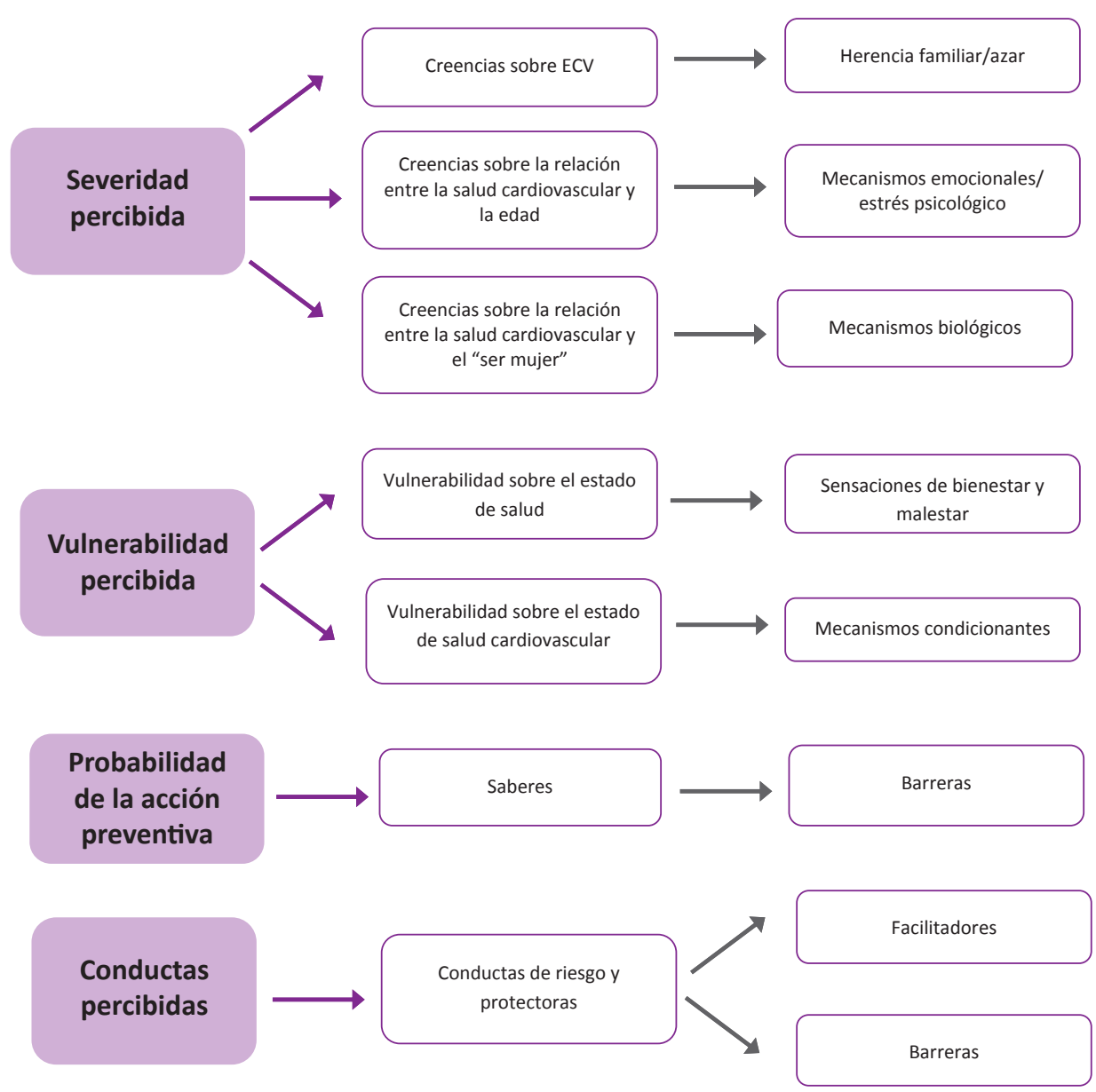

Fuente: Elaboración propia en base a las dimensiones del MCS y categorías emergentes.

Figura 1. Marco conceptual de subjetividades.

de riesgo, haciendo mención a que estas enfermedades eran el colesterol, tiroides, sobrepeso, diabetes o el hábito de consumir tabaco.

"Colesterol por las arterias...No sé qué otras..." (mujer, 49 años, hipertensa-Programa preventivo de actividad física).

\section{Conductas percibidas}

Cuando se indagó sobre las percepciones de las mujeres en torno a ciertas conductas de riesgo o protección en torno a las ECV se observó un cierto grado de conocimiento en ambos grupos analizados.

Las conductas se enfocaron en el colesterol elevado en sangre y en menor medida el sedentarismo y la alimentación poco saludable. Por otra parte, se visualizó muy poca percepción sobre otros factores como el consumo de sustancias tóxicas (tabaco o alcohol), obesidad, y/o la presión arterial.

Cabe resaltar que, el factor de riesgo "sedentarismo" emergió principalmente de los discursos de las mujeres que se encontraron formando parte del programa preventivo basado en actividad física, constituyéndose en un elemento facilitador.

Otro dato distintivo es que, si bien las mujeres pudieron identificar algún/os factor/es de riesgo para ECV, se observó que algunas partían del supuesto que sí conocían la existencia de estos factores, pero al momento de identificaros y profundizar sobre estos presentaron ciertas barreras.

Gran parte las entrevistadas no supieron reconocer ninguna conducta que beneficie el cuidado de la salud cardiovascular, mientras que las que sí reconocieron la existencia, solo hicieron alusión a la actividad física y a la alimentación saludable.

\section{DISCUSIÓN}

Desde este estudio se encontró que el grupo de mujeres percibió su estado de salud general y cardiovascular como "bueno", con cierto grado de desconocimiento sobre cuáles son las ECV y las conductas que benefician el cuidado de la salud cardiovascular y con creencias sobre el origen de estas enfermedades vinculadas a herencia familiar o azar. El factor de 
riesgo sedentarismo fue reconocido principalmente por mujeres bajo programa preventivo y no se visualizaron creencias sobre el vínculo entre la "edad" y "ser mujer" con las ECV. El poco conocimiento encontrado sobre las ECV fue similar a lo hallado en otros estudios realizados en el grupo de mujeres a pesar de las diferencias socioculturales de los grupos estudiados ${ }^{[23]}$.

Aunque las informantes en este trabajo poseían una percepción o comprensión sobre algunas conductas de riesgo para las ECV se observó que confundían, dudaban o presentaban cierta dificultad de profundizar sobre estos aspectos. Sin embargo, estos resultados difieren de los obtenidos en otro estudio realizado en el país ${ }^{[15]}$, probablemente porque las políticas locales o las intervenciones de prevención de ECNT no hacen foco hacia las ECV.

El rol del sedentarismo dentro de los factores de riesgo se encontró de manera más marcada en las mujeres que formaron parte del programa preventivo basado en la práctica de actividad física, llevando a resaltar los potenciales beneficios de los programas preventivos en salud pública al momento de adquirir conocimientos, percibir cambios y/o beneficios y posteriormente la posibilidad de plasmarlos en prácticas concretas de cuidados a la salud.

Cuando se buscó establecer posibles vínculos entre la salud cardiovascular y el "ser mujer" o transitar la vida adulta, emergieron del análisis algunos elementos de desconocimiento, siendo estos similares a los obtenidos en otros estudios nacionales y del cono sur ${ }^{[13,15]}$.

Además, la vinculación entre el género y las ECV fueron anclados a modos de subjetivación de género femenino tradicional, donde las mujeres tendrían una tendencia a "recargarse" en la edad adulta, aumentando su vulnerabilidad coronaria, es decir que en este campo subjetivo cobran relevancia aspectos afectivoemocionales o el estrés psíquico que vienen asociados a vivencias y problemáticas que se presentan de manera frecuente en la cotidianeidad del paso por la vida adulta, siendo estos elementos concordante con los reportados en otros contextos ${ }^{[12,24]}$ y donde se pone también en evidencia al igual que en este trabajo que la "edad" es uno de los predictores subjetivos de severidad cardiovascular menos percibidos ${ }^{[25]}$.

Otro resultado a destacar se da en torno a las percepciones sobre el estado de salud general y salud cardiovascular, donde la mayoría de las mujeres las manifestó como "bueno" a pesar de tener algún/os factor/es de riesgo ya adquirido/s; es decir no perciben de manera clara elementos subjetivos en torno a la dimensión de vulnerabilidad de enfermar.

Estos resultados cualitativos también fueron detectados en un grupo de mujeres de Australia, donde se observó que ellas subestimaron su riesgo de ECV, enfrentando muchas barreras al momento de reducir sus riesgos ${ }^{[25]} y$ donde a pesar de que posean un buen caudal de conocimiento sobre los factores de riesgo cardiovasculares la percepción del riesgo asociado a estos es baja ${ }^{[26]}$.
Pocos saberes sobre factores que protegen o ponen en riesgo la salud cardiovascular más una baja percepción de riesgo podría llevar probablemente a un grado bajo de preparación o disposición psicológica para plasmar una determinada acción preventiva de cuidado a la salud.

Se pudo contactar que en la construcción simbólica de las mujeres sobre las enfermedades cardiovasculares se encontraron vinculadas a imaginarios que se trasmiten solamente a través de "herencia familiar" o meramente por "azar". Estos resultados son similares a los hallazgos en un estudio de percepciones en un grupo de varones coronarios donde se detectaron algunas reflexiones a que el infarto "era algo que les podía pasar" [6].

Este estudio presenta algunas limitaciones propias del abordaje metodológico. En primer lugar, se trata de un estudio exploratorio cualitativo donde los resultados no son representativos, generalizables y extrapolables a otros grupos sociales que asisten al sistema público, a otros destinatarios de programas preventivos y/o aplicable a otros contextos. Sin embargo, desde los diseños cualitativos se busca explicar y lograr aproximaciones de manera inductiva y local a un fenómeno social determinado. En segundo lugar, no se seleccionaron informantes del género masculino, por lo que no se pudo lograr comparaciones de resultados entre hombres y mujeres que permitan una visión más amplia e inclusiva desde la perspectiva de género dentro del abordaje de las ECV y en el marco de las intervenciones en salud pública.

No obstante, la evidencia actual expone ciertas asimetrías en algunas sociedades entre hombres y mujeres, donde estas últimas tienen menos acceso a la información, atención sanitaria, y a los servicios y recursos necesarios para proteger su salud ${ }^{[10]}$.

Sin embargo, estos resultados resultan valiosos para proponer nuevas líneas de acción e investigación y poder contribuir a la producción de evidencia desde la esfera cualitativa que complemente a los datos cuantitativos ya existentes en el país sobre la prevalencia e incidencia de factores de riesgos y poder pensar las acciones desde los sistemas y políticas de salud pública disminuyendo las brechas entre los géneros.

Se puede concluir a partir de explorar diferentes dimensiones desde la perspectiva de un grupo de mujeres de La Pampa, Argentina, que existe algún grado de desconocimiento y una baja percepción de riesgo reforzados por ciertas creencias arraigadas sobre las ECV que pueden además estar explicadas por diferentes causas sociales, culturales y de género. Además, se identificó la presencia de barreras y facilitadores desde la esfera psicosocial que deberían ser consideradas al momento de pensar intervenciones de prevención de ECV desde un enfoque de género.

\section{Agradecimientos:}

Agradecemos a todas las mujeres que formaron parte de este estudio. 
Fuente de financiamiento: Las autoras declaran no haber recibido ninguna financiación para la realización de este trabajo.

Declaración de conflicto de intereses: Los autores declaran no tener conflicto de intereses con la publicación de este artículo.

* Los resultados del presente estudio contribuyeron a la etapa formativa de un proyecto de intervención a nivel local en el marco del "Programa Nacional Municipios y comunidades Saludables" del Ministerio de Salud de la Nación de Argentina.

\section{REFERENCIAS BIBLIOGRÁFICAS}

1. Organización Mundial de la Salud. Informe sobre la situación mundial de las enfermedades no transmisibles 2014 [Internet]. Ginebra: OMS; 2014 [citado el 20 de noviembre de 2016]. Diponible en: http://apps.who.int/iris/bitstream/10665/149296/1/ WHO_NMH_NVI_15.1_spa.pdf?ua=1\&ua=1

2. World Health Organization. The Global Burden of Disease [Internet]. Geneva: WHO; 2004 [citado el 10 de noviembre de 2016]. Diponible en: http://www.who.int/healthinfo/ global_burden_disease/GBD_report_2004update_full.pdf

3. World Health Organization. Concommunicable Diseases (NCD) Country Profiles. Health statistics and health information systems [Internet]. Geneva: WHO; 2014 [citado el 2 de noviembre de 2016]. Diponible en: http://www.who.int/nmh/publications/ ncd-profiles-2014/en/

4. Ministerio de Salud de la Nación, Dirección de Estadísticas e Información. Estadísticas Vitales. Información Básica Año 2009 [Internet]. Buenos Aires: Ministerio de Salud; 2009 [citado el 5 de octubre de 2016]. Disponible en: http://www.deis.gov.ar/ Publicaciones/Archivos/Serie5Nro53.pdf

5. Instituto Nacional de Estadística y Censos. Tercera Encuesta Nacional de Factores de Riesgo 2013 [Internet]. Buenos Aires: INDEC; 2013 [citado el 25 de octubre de 2016]. Disponible en: http://www.indec.gob.ar/ftp/cuadros/menusuperior/enfr/ doc_base_usuario_enfr2013.pdf

6. Tajer D, Reid G, Gaba M, Lo Russo A, Barrera MI. Investigaciones sobre género y determinación psicosocial de la vulnerabilidad coronaria en varones y mujeres. Rev Argent Cardiol. 2013;8(4):344-52.

7. Mozaffarian D, Benjamin EJ, Go AS, Arnett DK, Blaha MJ, Cushman M, et al. Heart disease and stroke statistics-2015 update: a report from the american heart association. Circulation. 2015;131(4):e29-e322.

8. Organización Mundial de la Salud. Determinantes Sociales de la Salud [Internet]. Ginebra: OMS; 2016 [citado el 15 de setiembre de 2016]; Disponible en: http://www.who.int/ social_determinants/es/

9. Buquet Corleto, AG. El orden de género en la educación superior: una aproximación interdisciplinaria. Nómadas. 2016;44:27-43.

10. Organización Panamericana de la Salud, Organización Mundial de la Salud. Estrategia para la prevención y el control de las enfermedades no transmisibles [Internet]. Washington, DC: OMS: 2012 [citado el 1 de octubre de 2016]. Disponible de: http://www.paho.org/hq/index.php?option=com_ docman\&task=doc_view\&gid=24045\&Itemid=270

11. The American Heart Association (AHA). Cardiovascular Disease: Women's No. 1 Health Threat [Internet]. Washington, DC: AHA; 2010 [citado el 1 de octubre de 2016]; Disponible en: https:// www.heart.org/idc/groups/heart-public/@wcm/@adv/ documents/downloadable/ucm_302256.pdf

12. Organización Mundial de la Salud. Estrategia para incorporar el análisis y las acciones de género en las actividades de la OMS [Internet]. Ginebra: OMS; 2009 [citado el 6 de setiembre de 2016]. Disponible en: http://www.who.int/gender/ documents/gender/9789241597708/es/

13. Artucio C, Duro I. Encuesta de percepción de la enfermedad cardiovascular de la mujer por la mujer. Rev Urug Cardiol. 2012;27(3):304-12

14. Coca, A. Cardiovascular disease based on gender: myths and evidence. Rev Clin Esp. 2012;212(2):81-3.

15. Rolandi F, Grinfeld L, Sosa Liprandi MI, Tambussi A, Kazelian L, et al. Encuesta de reconocimiento de la enfermedad cardiovascular en la mujer por la mujer. Rev. argent. cardiol. 2013;81(4):329-335.

16. Maxwell J. Qualitative Research Design. An Interactive Approach. Londres: Sage Publications; 1996.

17. Becker $\mathrm{MH}$. The health belief model and personal health behavior. Thorofare, NJ: Charles B. Slack; 1974.

18. Rosenstock IM. Historical Origins of the Health Belief Model. Health Education Monographs. 1974;2(4):328-35.

19. Flick U. Introducción a la investigación cualitativa: Estrategias de muestreo. Madrid: Ediciones Morata; 2004.

20. Valles M. Entrevistas cualitativas. Madrid: Centro de Investigaciones Sociológicas; 2007.

21. Hostil OR. Content analysis for the social sciences and humanities. New York: Addison Wesley; 1968.

22. Fernandez RA, Del Sueldo R, Vicario A, Del Sueldo MA, Cerezo G, Enders JE. Estudio de los factores de riesgo cardiovascular y el estado cognitivo en mujeres adultas de la ciudad de Villa María, Córdoba y Rancul, La Pampa. Buenos Aires: Comisión Nacional Salud Investiga del Ministerio de Salud de la Nación; En prensa.

23. Querales M, Ruiz N, Rojas S, Espinoza M. Nivel de conocimiento sobre factores de riesgo cardiovascular en una comunidad de Naguanagua, Venezuela. Rev Salud Pública. 2011;13(5):759-71.

24. Oertelt-Prigione S, Seeland U, Kendel F, Rucke M, Floel A, Gaissmaier $\mathrm{W}$, et al. Cardiovascular risk factor distribution and subjective risk estimation in urban women--the BEFRI study: a randomized crosssectional study. BMC Med. 2015;13:52.

25. Gholizadeh L, Digiacomo M, Salamonson Y, Davidson PM. Stressors influencing Middle Eastern women's perceptions of the risk of cardiovascular disease: a focus group study. Health Care Women Int. 2011;32(8):723-45.

26. Perez-Manchon D, Alvarez-Garcia GM, Gonzalez-Lopez E. [Perception of cardiovascular risk in an outpatient population of the Madrid Community]. Hipertens Riesgo Vasc. 2015;32(3):100-4. 